\title{
Enteroparasitoses em gestantes e puérperas no Rio de J aneiro
}

\author{
Enteroparasitosis in pregnant and post-partem \\ women in Rio de J aneiro
}

Lêda Maria da Costa Macedo 1

Luis Rey 2

\footnotetext{
1 Departamento de Parasitologia, Universidade do Estado do Rio de Janeiro. Rua Teodoro da Silva 48, 50 andar, Rio de Janeiro, RJ 20551-001, Brasil.

2 Departamento de Medicina

Tropical, Instituto Oswaldo Cruz, Fiocruz. C.P. 926 , Rio de Janeiro, RJ 21045-900, Brasil.
}

Abstract From November 1989 to May 1991, in Rio de Janeiro, Brazil, the prevalence of intestinal parasitosi s was investigated in 795 women coming from the Public Health Services, who were divided into three groups: 490 pregnant women in pre-natal care period, 104 after-delivery women and 201 non-pregnant women (control). Coproparasitologic tests were run using the Sedimentation through Centrifugation, Faust, Kato-Katz and Baermann-Moraes methods. Positivities were found ranging from $37.6 \%$ to $53.6 \%$ for pregnant women, $38.3 \%$ for non-pregnant women and $69.2 \%$ for after-delivery women, with statistically significant differences between the rates of pregnant women and after-delivery women, and with no statistic difference between the groups of pregnant and non-pregnant women. The most frequent parasites found were A. Iumbricoides and T. trichiura. The conclusion was that the control of intestinal parasitosis is not a priority in the pregnant population studied because, in spite of the regular assistance provided during the pre-natal care period, they keep on infected .

Key words Intestinal Parasites; Pregnant Women; Ascaris Iumbricoides; Trichuris trichiura; Epidemiology

Resumo A prevalência de parasitoses intestinais foi determinada em 795 mulheres distribuídas em grupos de 490 gestantes, 201 não-gestantes (controle) e 104 puérperas, no período de novembro de 1989 a mai o de 1991, no Rio de Janei ro, Brasil. Exames coproparasi tológi cos foram realizados pel os métodos de Sedimentação por Centrifugação, Faust, Kato-Katz e Baermann-Moraes. A prevalência de enteroparasitoses entre os grupos de gestantes $(37,6 \%)$ e o grupo-controle (38,3\%) pelo método de sedimentação por centrifugação foi praticamente igual, apesar de estar o primei ro grupo regularmente assi sti do por serviços especializados de saúde. Ascaris lumbricoides e Trichuris trichiura foram os parasitos mais freqüentes (em torno de $30,0 \%$ ). Quando métodos mais específicos foram aplicados em 168 amostras de gestantes, o percentual foi el evado para 53,6\%. Houve diferença estatística si gnificati va entre a taxa de gestantes e puérperas $(69,2 \%)$. Estes achados mostram a pouca atenção dispensada a estas infecções durante a gestação, não obstante relatos anteriores das possí vei s repercussões para o feto e o recém-nasci do.

Palavras-chave Parasitos Intestinais; Gestantes; Ascaris lumbricoides; Trichuris trichiura; Epidemiologia 


\section{Introdução}

São comuns os debates realizados sobre saúde no Rio de Janeiro, onde representantes dos Conselhos de Medicina e de outros órgãos públicos, e às vezes com a participação até do Ministro da Saúde, testemunham o constrangimento que hoje enfrenta quem necessita procurar a rede pública de saúde para atendimento. Desta forma, em relação à assistência materno-infantil, verifica-se que o atendimento usualmente acontece de modo segmentado e descontínuo, sendo precários os cuidados básicos de saúde dispensados a este grupo tão intimamente relacionado.

No que se refere às enteroparasitoses, embora altas prevalências sejam detectadas muito freqüentemente nos países em desenvolvimento, pouco se conhece sobre o curso da infecção parasitológica intestinal na gestante, ou sobre as possíveis repercussões dessas infecções para o desenvolvimento fetal, conforme refere Brabin (1985). A associação entre gravidez e parasitoses tem despertado o interesse de alguns investigadores, por se tratar de grupo com características próprias e transitórias dos padrões metabólicos, endocrinológicos e imunológicos.

Quanto à prevalência das enteroparasitoses durante a gestação, as taxas são bastante variáveis, dependendo do local pesquisado, sendo que o Ascaris lumbricoides, os ancilostomatídeos e o Trichuris trichiura são as espécies mais encontradas, segundo Constantine et al. (1988), D'Alauro et al. (1985), Ezeoke (1986), Guerra et al. (1991), Roberts et al. (1985), St. George (1976) eVillar et al. (1989).

Os objetivos deste estudo foram estabelecer a prevalência das enteroparasitoses em gestantes, não-gestantes (controle) e puérperas, e discutir a necessidade de aten ção médica destinada ao controle das parasitoses intestinais nos grupos acima mencionados.

\section{Material e métodos}

População estudada

Foram estudadas 795 mulheres com faixa etária entre 15 e 34 anos, distribuídas nos seguintes grupos:

Gestantes: Grupo de 490 gestantes em acompanhamento pré-natal, sendo 168 oriundas de Maternidade Municipal (MM), e 322 do Posto de Atendimento Médico (PAM) Federal, ambos localizados no Rio de Janeiro. Os potes para a coleta de material foram distribuídos às mu-
Iheres como parte da rotina dos exames realizados durante o pré-natal. As amostras de fezes foram coletadas nos períodos de novembro de 1989 a setembro de 1990 e de março a maio de 1991, respectivamente.

Grupo-controle: Grupo oriundo do PAM no período de março a maio de 1991, constituído de 201 mulheres que eram mães de crianças acompanhadas no Serviço de Puericultura do PAM durante este período. Foram selecionadas as mães de crianças menores de dois anos, que haviam realizado pré-natal na última gravidez, e que não estavam grávidas nem eram puérperas no momento da pesquisa. Após entrevista, foram entregues os potes com orientação para a coleta de material.

Puérperas: Do grupo de 230 puérperas internadas em alojamentos conjuntos (AC) da MM no período de junho de 1988 a setembro de 1990, foram obtidos dados sobre o local de realização do pré-natal. Cada puérpera recebeu recipiente para a coleta de fezes frescas, o qual era recolhido após 24 a 48 horas.

Aos três grupos perguntamos sobre a presença de infecções parasitológicas prévias ao estudo.

\section{Métodos coproparasitológicos}

Foram empregados os seguintes métodos de concentração: 1) Técnica de Sedimentação por Centrifugação em sistema descartável, constituído de tubo cônico ( $15 \mathrm{ml}$ ) e funil com extremidade porosa que funciona como filtro (PARASITOKIT, Biotécnica). O material foi diluído em água, filtrado e centrifugado a 1.500 rpm durante dois minutos; 2) Técnica de Centrífugo - flutuação em Sulfato de Zinco segundo Faust et al. (1939); 3) Técnica de Kato-Katz por Katz et al. (1972); 4) Técnica de Baermann-Moraes, conforme Moraes (1948).

A técnica de sedimentação por centrifugação foi realizada nos laboratórios das instituições de atendimento (MM e PAM), enquanto que os outros métodos foram executados no laboratório da Disciplina de Parasitologia da Faculdade de Ciências Médicas da Universidade do Estado do Rio de Janeiro (FCM-UERJ).

\section{Tratamento estatístico}

A escala nominal caracterizou o nível de mensuração das amostras tabuladas. O Qui-Quadrado foi o teste indicado para a análise das tabelas, sendo considerado o nível de significância de 95,0\% (Siegel, 1975). 


\section{Resultados}

\section{Gestantes e grupo-controle}

As positividades encontradas nos grupos de gestantes estudados não diferiram estatisticamente, mostrando uma taxa média de infecção de $37,1 \%$ das mulheres, quando utilizada apenas uma técnica parasitológica (sedimentação por centrifugação). Para o grupo-controle, também não houve diferença significativa quando comparado com gestantes do mesmo local, ambos apresentando freqüências praticamente idênticas (Tabela 1).

Com relação aos parasitos encontrados, o A. lumbricoi des foi o mais prevalente em gestantes e não-gestantes, com freqüência média de $16,1 \%$. A distribuição das taxas de positividade entre os geo-helmintos encontrados nos três grupos não mostrou diferença significativa entre eles, exceto para o S. stercoralis, que foi mais freqüente entre as gestantes do PAM (Tabela 1).

\section{Gestantes e puérperas}

A utilização de três métodos específicos de detecção de parasitos em 104 amostras de puérperas e 168 amostras de gestantes determinou uma positividade de $69,2 \%$ e $53,6 \%$, respectivamente, com diferença estatisticamente significativa (Tabela 2 )

Com relação ao mono e ao poliparasitismo, a distribuição das espécies entre os grupos de gestantes e puérperas não mostrou diferença, o mesmo sucedendo com cada espécie, exceto ancilostomatídeos. Entre as puérperas, também os parasitos mais prevalentes foram $\mathrm{T}$. trichiura e A. Iumbricoides, sendo o segundo responsável pela infecção em mais de um quarto das pacientes (Tabela 2).

Quanto aos protozoários intestinais, consideramos E. histolytica todo cisto encontrado apresentando até quatro núcleos. Não foi objeto do nosso estudo a diferenciação morfométrica dos cistos.

No que diz respeito à realização de consultas pré-natais durante a gestação, obtivemos informações de 187 puérperas (81,3\%). Constatamos que apenas 27 fizeram pré-natal na própria instituição (14,4\%), 94 em outros serviços (50,3\%) e 66 (35,3\%) não o fizeram ou o dado era desconhecido.

Na distribuição das enteroparasitoses segundo o conhecimento da mulher sobre o parasitismo prévio, verificamos que a prevalência encontrada foi independente da informação fornecida pela paciente (Tabela 3).
Tabela 1

Distribuição de enteroparasitos nos grupos de 168 gestantes da Maternidade Municipal (MM), 322 do Posto de Atendimento Médico (PAM) e 201 não-gestantes (PAM), pelo método de sedimentação por centrifugação, RJ , 1991.

\begin{tabular}{lccc}
\hline Enteroparasitos & $\begin{array}{c}\text { Gestantes (MM) } \\
\text { \% de positividade }\end{array}$ & $\begin{array}{c}\text { Gestantes (PAM) } \\
\text { \% de positividade }\end{array}$ & $\begin{array}{c}\text { Não-gestantes } \\
\text { \% de positividade }\end{array}$ \\
\hline Ascacris lumbricoides & 18,4 & 15,5 & 14,4 \\
Trichuris trichiura & 4,2 & 7,8 & 4,5 \\
Ancilostomatídeo & 1,2 & 1,2 & 1,5 \\
Strongyloides stercoralis & $0,6 *$ & $5,0 *$ & 3,0 \\
Enterobius vermicularis & 0,6 & - & - \\
Giardia lamblia & 2,4 & 4,0 & 5,5 \\
Entamoeba coli & 9,5 & 9,0 & 7,0 \\
Entamoeba histolytica & 2,4 & 1,9 & 1,0 \\
Endolimax nana & 5,4 & 5,9 & 9,5 \\
lodamoeba butschlii & - & 1,2 & 1,5 \\
Total & $35,7^{* *}$ & $38,5^{* *}$ & $38,3 * *$
\end{tabular}

* Significativo $\mathrm{p}<0,05$

** Diferença não significativa

Tabela 2

Distribuição dos enteroparasitos em 168 gestantes e 104 puérperas da Maternidade Municipal (MM), utilizando os métodos de Kato-Katz, Faust e Baermann-Moraes, RJ , 1991.

\begin{tabular}{lcc}
\hline Enteroparasitos & $\begin{array}{c}\text { Gestantes } \\
\text { de positividade }\end{array}$ & $\begin{array}{c}\text { Puérperas } \\
\text { de positividade }\end{array}$ \\
\hline Ascacris lumbricoides & 25,6 & 32,7 \\
Trichuris trichiura & 28,6 & 37,5 \\
Ancilostomatídeo & $5,4^{*}$ & $15,4^{*}$ \\
Strongyloides stercoralis & 4,8 & 9,6 \\
Enterobius vermicularis & 0,6 & - \\
Giardia lamblia & 2,4 & 1,9 \\
Entamoeba coli & 14,3 & 9,6 \\
Entamoeba histolytica & 1,2 & 1,0 \\
Endolimax nana & 7,1 & 6,7 \\
lodamoeba butschlii & 1,2 & 1,9 \\
Total & $53,6 *$ & $69,2 *$
\end{tabular}

* Significativo $\mathrm{p}<0,05$

Tabela 3

Distribuição dos enteroparasitos entre 374 mulheres incluídas no estudo, segundo o conhecimento delas sobre infecção parasitária prévia à pesquisa.

\begin{tabular}{lcc}
\hline $\begin{array}{l}\text { Respostas das mulheres } \\
\text { sobre parasitose prévia }\end{array}$ & $\begin{array}{c}\text { Mulheres entrevistadas } \\
n\end{array}$ & $\begin{array}{c}\text { positividade* } \\
\%\end{array}$ \\
\hline Não sabem & 179 & 64,2 \\
Têm & 73 & 68,5 \\
Não têm & 122 & 54,9 \\
\hline
\end{tabular}

* Diferenças não significativas 


\section{Discussão}

As parasitoses intestinais em gestantes têm sido referidas apenas quando ocorrem casos isoIados com complicações clínicas, como nos trabalhos de Cintron-Villaronga (1967), King (1929), Oliveira (1947) e Russel-Corfield \& Hirson (1922). Autores como D'Alauro et al. (1985) e Roberts et al. (1985) não detectaram alterações em gestantes parasitadas quanto à freqüência de prematuridade ou quanto ao pesomédio dos recém-nascidos. Para St. George (1976), entretanto, esta correlação foi estabelecida. Villar et al. (1989), analisando 14.914 gestantes, verificaram aumento significativo na incidência de retardamento de desenvolvimento intra-uterino em mulheres infectadas com A. lumbricoidese T. trichiura. Esta associação foi dependente da carga parasitária e não foi observada para Strongyloides stercoralis. Mac Leod (1988) admite para o A. lumbricoides e os ancilostomatídeos a interferência do parasitismo no curso da gravidez, com possíveis repercussões para o feto e o recém-nascido.

Diante da incerteza sobre as conseqüências do parasitismo intestinal na gravidez e dos poucos estudos encontrados sobre essa associação, o tratamento antiparasitário tem sido feito segundo critérios adotados individualmente por cada profissional. Além disso, pouco se conhece sobre as repercussões das drogas anti-hel mínticas para o desenvolvimento do feto. Enquanto autores como D'Alauro et al. (1985) e Roberts et al. (1985) não recomendam o tratamento de parasitoses durante a gestação nos casos de parasitismo assintomático ou de sintomas leves, Barua \& Foll (1960) e Oliveira (1947) o preconizam a todas as gestantes parasitadas. Roberts et al. (1985) fazem a observação de que o tratamento antiparasitário deva ocorrer antes da gravidez, com o que Mac Leod (1988) concorda e sugere que essa ação deva fazer parte de programas de saúde materna.

Na mulher, a gestação, o parto e o puerpério são fases que, embora vividas por uma mesma pessoa, geralmente não são acompanhadas pelos mesmos profissionais, sendo tratadas de forma isolada nos serviços públicos de saúde no Rio de Janeiro. Profissionais que atendem a cada uma dessas fases freqüentemente ignoram a evolução clínica da paciente nas outras.

A falta de continuidade no acompanhamento das gestantes se torna bem clara quando anal isamos a questão das enteroparasitoses. Embora a classe mais carente da população brasileira seja também a mais parasitada e a que mais se utiliza dos serviços públicos de Saúde, não existe no momento nenhum proje- to de controle de enteroparasitoses na gestação, mesmo que no nível individual ou familiar, durante o acompanhamento pré-natal. Para esta fase, inclusive, não há padronização da conduta médica relativa à gestante parasitada. Administrativamente, o exame de fezes nem sequer é exigido pelo Ministério da Saúde (1988); as normas editadas para a assistência pré-natal preconizam este exame apenas nos casos com sintomatologia gastrintestinal. A Secretaria Municipal de Saúde do RJ (1984), por outro lado, inclui em suas normas o coproparasitológico como de rotina na assistência prénatal. A pouca atenção dada ao resultado de um exame parasitológico na gestação explica por que a alta taxa de parasitismo entre as gestantes, que estão sob acompanhamento médico, não difere das de mulheres do grupo-controle, que não contam com supervisão médica regular.

Somam-se a isto as dificuldades de tratamento dos parasitos intestinais em relação aos medicamentos de uso mais popular (benzimidazólicos). No caso do Mebendazol, medicamento comumente utilizado nas geo-helmintíases por ser polivalente, eficaz e de baixo custo, de acordo com os relatos de M ac Leod (1988) e da Organização Mundial da Saúde (1991), faltam dados sobre a passagem transplacentária da droga e sua eliminaçäo no leite materno, o mesmo acontecendo com o Albendazol, droga ainda não liberada pelo F.D.A. (Food and Drug Administration-USA), mas bastante usada no nosso país. Fica por conta de critérios individuais médicos o tratamento das enteroparasitoses, que, quando prescrito, deverá acontecer sempre após o primei ro trimestre de gestação, conforme preconiza a Organização Mundial da Saúde (OMS, 1991).

Dados sobre a prevalência de parasitoses intestinais na população gestante ou inexistem ou são subestimados por conta da precariedade no diagnóstico parasitológico. Daí, a baixa positividade encontrada em vista do uso de técnicas parasitológicas pouco sensíveis, ou da falta de profissionais treinados em leitura coprológica. O fato de termos encontrado cerca de $53,6 \%$ de gestantes parasitadas é razão suficiente para que se organize o tratamento das pacientes, e se acompanhem os casos, quanto às possíveis repercussões das drogas quanto à toxicidade, teratogenicidade e outros efeitos colaterais.

A diferença quanto às técnicas empregadas fica evidente quando as taxas de infecção encontradas com o emprego de apenas um método, como fazem em geral os laboratórios públicos, são comparadas com as elevadas taxas ob- 
tidas quando técnicas mais específicas são aplicadas. Nossos resultados não parecem indicar que a gravidez tenha influído sobre a inci dência de cada parasitose em particular .

Os dados que obtivemos na Tabela 3 aponta para uma total desinformação da mulher quanto à possível infecção parasitária intestinal prévia, traduzindo em informações não fidedignas por parte delas. Podemos considerar as hipóteses dessas infecções serem pouco importantes para os profissionais de saúde e/ ou para os portadores, além da possibilidade desses profissionais, apesar de valorizarem os resultados dos exames parasitológicos, não transferirem as informações para as pacientes.

As diferenças encontradas nas positividades entre gestantes e puérperas da mesma instituição podem ser explicadas pelo fato de apenas $14,4 \%$ das gestantes terem feito seus partos na própria instituição, o que prejudica a comparabilidade das duas populações.

A conseqüência é que, embora as mulheres durante a gravidez procurem auxílio médico, objetivando a busca e manutenção do seu bem- estar físico e de sua prole, passam simplesmente de gestantes parasitadas a mães parasitadas.

Embora o número de gestantes examinado tenha sido facilitado pela rotina dos exames complementares solicitados pelo médico durante as primeiras consultas no pré-natal, em se tratando de puérperas, este inquérito foi bastante difícil. A não conscientização materna e da equipe de saúde sobre a importância do exame parasitológico fez com que menos da metade das puérperas colhesse material para exame. Outros fatores, como a lavagem intestinal pré-parto, também contribuíram para a baixa amostragem, por levarem a paciente ao retorno das evacuações cerca de 48 horas após o parto, muitas vezes coincidindo com a alta hospitalar.

Os dados acima mostram a necessidade de se diagnosticar, informar e tratar efetivamente as gestantes; ministrar educação sanitária às mães de forma a prevenir sua reinfecção e estudar drogas e esquemas de tratamento das enteroparasitoses mais adequados a esses grupos analisados.

\section{Referências}

BARUA, R. N. \& FOLL, C. V., 1960. Anaemias of pregnancy in upper Assam and upper Burma. Journal of Tropical Medicineand Hygiene, 63:225-232.

BRABIN, B. J., 1985. Epidemiology of infection in pregnancy. Reviews of Infectious Diseases, 7:579-603.

CINTRON-VILLARONGA, J. R., 1967. Ascaris and Uncinaria in pregnancy. Boletin de la Associacion Médica de Puerto Rico, 59:468-471.

CONSTANTINE, G.; ARUNDELL, L.; FINNN, K.; LOWE, P.; O'CONNOR, A. \& LUESLEY, D. M., 1988. Helminth infestations in asian women attending an antenatal clinic in England. British Journal of Obstetrics and Gynaecology, 95:493-496.

D'ALAURO, F.; LEE, R. V.; PAO-IN, K. \& KHAIRALLAH, M., 1985. Intestinal parasites and pregnancy. Obstetrics and Gynecology, 66:639-643.

EZEOKE, A. C. J., 1986. Helminthic infections and some haematological parameters in pregnant women in Calabar (Nigeria). Revista Ibérica deParasitologia, 46:123-128.

FAUST, E. C.; SAWITZ, W.; TOBIE, J.; ODOM, V.; PERES, C. \& LINCICOME, D. R., 1939. Comparative efficiency of various technics for the dignosis of protozoa and helminths in feces. Journal of Parasitology, 25:241-262.
GUERRA, E. M.; VAZ, A. J.; TOLEDO, L. A. S.; IANONI, S. A.; QUADROS, C. M. S.; DIAS, R. M. D. S. \&BARRETO, O. C. O., 1991. Infecções por helmintos e protozoários intestinais em gestantes de primeira consulta atendidas em centros de saúde da rede estadual no Subdistrito do Butantã, Município de São Paulo. Revista do Instituto de Medicina Tropical deSão Paulo, 33:303-308.

KATZ, N., CHAVES, A. \& PELLEGRINO, J., 1972. A simple device for quantitative stool thick-smear technique in schistosomiasis mansoni. Revista do Instituto de Medicina Tropical de Säo Paulo, 14:397-400.

KING, E. L., 1929. Hookworm disease and pregnancy. American Journal of Obstetrics and Gynecology, 18:569-575.

MAC LEOD, C. L., 1988. Intestinal nematodes. In: Parasitic Infections in Pregnancy and the Newborn. (C. L. Mac Leod, org.), pp. 192-215, New York: Oxford University Press.

MORAES, R. G., 1948. Contribuição para o estudo do Strongyloides stercoral is e da estrongiloidose no Brasil. Revista do Serviço Especial de SaúdePública, 1:507-624. 
OLIVEIRA, G. G., 1947. Toxemias pseudo-gravídicas e parasitoses intestinais. Arquivos de HigieneeSaúdePública, 12:107-111.

OMS (Organizacion Mundial de la Salud), 1991. ModeIo OMS de Información sobre Prescripción de Medicamentos: Medicamentos Utilizados en las Enfermidades Parasitárias. Genebra: OMS.

ROBERTS, N. S.; COPEL, J. A.; BHUTANI, V.; OTIS, C. \& GLUCKMAN, S., 1985. Intestinal parasites and other infections during pregnancy in southeast asian refugees. Journal of Reprodutive Medicine, 30:720-725.
RUSSEL CORFIELD, C. \& HIRSON, B., 1922. A case of acute ascariasis associated with T. trichiura complicating pregnancy. The Lancet, 1:995-996.

SIEGEL, S., 1975. Estatística Não Paramétrica. São Paulo: Mc Graw Hill.

St. GEORGE, J., 1976. Intestinal parasitic infestation among parturients in Trinidad and Tobago. International Surgery, 61:222-225.

VILLAR, J., KLEBANOFF, M. \& KESTLER, E., 1989. The effect on fetal growth of protozoan and helminthic infection during pregnancy. Obstetrics and Gynecology, 74:915-920. 